\title{
DETERMINING FUNCTIONAL RELIABILITY OF PYROTECHNIC MECHANICAL DEVICES
}

\author{
Laurence J. Bement* \\ NASA Langley Research Center \\ Hampton, Virginia \\ Herbert A. Multhaup \\ New England, North Dakota
}

\begin{abstract}
This paper describes a new approach for evaluating mechanical performance and predicting the mechanical functional reliability of pyrotechnic devices. Not included are other possible failure modes, such as the initiation of the pyrotechnic energy source. The requirement of hundreds or thousands of consecutive, successful tests on identical components for reliability predictions, using the generally accepted go/no-go statistical approach routinely ignores physics of failure. The approach described in this paper begins with measuring, understanding and controlling mechanical performance variables. Then, the energy required to accomplish the function is compared to that delivered by the pyrotechnic energy source to determine mechanical functional margin. Finally, the data collected in establishing functional margin is analyzed to predict mechanical functional reliability, using small-sample statistics. A careful application of this approach can provide considerable cost improvements and understanding over that of go/no-go statistics. Performance and the effects of variables can be defined, and reliability predictions can be made by evaluating 20 or fewer units. The application of this approach to a pin puller used on a successful NASA mission is provided as an example.
\end{abstract}

\section{$\underline{\text { INTRODUCTION }}$}

Although pyrotechnics are required to perform critical aerospace mechanical functions, currently accepted test methods do not establish functional margins, nor are analyses statistically rigorous. As described in reference 1 , few guidelines exist for the application of pyrotechnics. The current approach is to treat these explosive and propellant-actuated

\footnotetext{
*Senior Pyrotechnic Engineer, Testing and Development Branch, Associate Fellow, AIAA

Copyright (C) by the American Institute of Aeronautics, Inc. No copyright is asserted in the United States under Title, U. S. Code. The U. S. Government has a royalty-free license to exercise all rights under the copyright claimed herein for Government Purposes. All other rights are reserved by the copyright owner.
}

mechanisms as "black boxes" without the ability to measure and quantify performance. The only quantifiable performance attribute is "go or no-go;" it either did or did not function as required. Thus, attribute testing is "blind" to any and all design variables (as well as tolerances on these variables), which actually control functional failures; indeed, attribute tests are blind to the very existence of any and all physics of failure. Over 30 years ago on the Gemini Program, mechanical functional margin requirements were imposed by conducting test firings with $+/-15 \%$ pyrotechnic loads. Although this requirement contributed nothing towards quantifying reliability predictions, it was introduced to provide a qualitative assurance that the devices would function properly. If it still functioned with an $85 \%$ load, some functional margin is implied. Or if it functioned without bursting with a $115 \%$ load, some structural containment margin is implied. As described in references 2 and 3, the $+/-15 \%$ testing does not quantitatively define either the relative effects of system parameters or actual mechanical functional margins. Consequently, when pyrotechnic mechanical system failures occur ${ }^{4}$, the most frequently cited cause of failures was a lack of understanding of pyrotechnic component and system functional mechanisms.

Personnel in the Halogen Occultation Experiment (HALOE) Project Office at NASA Langley Research Center were faced with just such a failure $^{2}$. A failure to properly stroke occurred in a newly manufactured lot (made under carefully traceable ingredients and controlled procedures) of pin pullers in a design that the project had selected for use on their mission. This lot of pin pullers was being manufactured by the same source, using the same drawings, as the units planned for use on HALOE. An investigation was conducted and a decision was made to redesign the pin puller, conduct another qualification, and predict the reliability of the redesigned pin puller.

The approach widely used in evaluating the reliability and confidence levels of pyrotechnically 
actuated mechanisms was to compile go/no-go functional statistics ${ }^{5}$. The basis for this approach is simply the number of tests conducted on samples of identical components; no functional measurements or evaluations are made. The following table from reference 5 shows the number of consecutively successful tests required to achieve two specific reliability levels at three specific confidence levels:

TABLE I. CONSECUTIVE SUCCESSES NEEDED TO ACHIEVE RELIABILITY USING GO/NO-GO TESTING

\begin{tabular}{ccc}
\hline \hline $\begin{array}{c}\text { Confidence } \\
\text { Level }\end{array}$ & $\begin{array}{c}99.9 \% \\
\text { Reliability }\end{array}$ & $\begin{array}{c}99.99 \% \\
\text { Reliability }\end{array}$ \\
\hline 50 & 700 & 7,000 \\
90 & 2,300 & 23,000 \\
95 & 3,000 & 30,000 \\
\hline \hline
\end{tabular}

Clearly, a requirement to increase either reliability or confidence level with this approach demands the testing of larger and larger quantities. Even at the lower reliability and confidence levels, such numbers are normally cost prohibitive.

When reliability and confidence level requirements are made in specifications, suppliers often first contend that the device they are offering has been "qualified" on another similar application. Then, they justify their ability to meet the reliability/confidence specification by referring to the number of successful tests they propose to accomplish on the lot to be manufactured for this particular application and on previous history. Typical manufacturing lots number less than 100 units, and, therefore, contribute little to realistic, quantified mechanical functional reliability. Lists of previous lots and applications of this device or "very similar, qualified" devices are provided, which implies that all previous hardware contributes toward the determination of reliability at some confidence level. Unfortunately, the statistical objective of using only identical units has been violated: 1) the historical samples tested are not from the same manufacturing lot, 2) previous designs often do not use the same materials, and 3) previous designs often do not meet the same "form, fit and function."

As an aside, statistical test methods, such as the Bruceton, Langlie and Probit, are used to determine the reliability of initiating pyrotechnic devices. The reliability predictions from these tests are frequently and erroneously used to imply the ability of the devices to accomplish intended mechanical functions. The capabilities to initiate and to accomplish a mechanical function are separate entities in the understanding of pyrotechnic performance. (The NASA Standard Initiator (NSI) is the component that has been demonstrated to best meet this critical initiation role). Only mechanical functional evaluations and reliability are addressed in this report.

The quandary for the potential user of pyrotechnics is that there is no currently accepted, standard procedure that provides a functional understanding of pyrotechnic mechanisms, while providing predictions of reliability for manufacturing lots to be used. Second, the cost of pyrotechnic devices prevents the use of large numbers for the accepted standard of go/no-go testing. Manufacturing lots usually number less than 100 units, often as few as 20. The purpose of this paper is to provide methodologies for determining both mechanical functional margins and the predicted reliability for accomplishing the intended task of pyrotechnically actuated mechanisms, using small samples. The following are specific objectives:

1. Use a performance-based evaluation logic to provide an understanding of functional mechanisms, variables and margins. Determine the most significant failure-controlling performance variable for analysis of reliability.

2. Use 2 to 20 functional tests from a manufacturing lot for final demonstration of mechanical functional reliability.

The determination of mechanical functional margin and reliability for the effort described in this paper was approached through the use of component and system-level performance data collected in the redesign, development and qualification of the HALOE pin puller. Tests were conducted to determine the energy required to accomplish the function, and to measure the energy delivered by the NSI (several different lots) in the pin puller. A final series of tests was conducted by test-firing five pin pullers in the HALOE Instrument to determine the performance of the pin puller in the flight system. The data collected were analyzed, using small-sample statistics, to predict the mechanical functional reliability of the units flown on the mission. 


\section{HARDWARE TESTED}

The hardware evaluated in this program were: (1) the NASA Langley Research Center HALOE pin puller, which was powered by (2) the NASA Standard Initiator (NSI).

\section{$\underline{\text { Pin Puller Description }}$}

The purpose of the redesigned HALOE pin puller, shown in figure 1 and reported in reference 2, was to withdraw the 0.25 -inch diameter pin into its body a distance of at least 0.563 inch to release a mechanical interface. A pin puller at each of two sites provided the mission-critical release of two gimbals, following the launch phase of the mission. Electrically initiating either of the NSIs, installed in the ports shown, drove the piston/pin from left to right. The hot gases from the NSI passed through a 0.10 -inch diameter opening in the bottom of the NSI port to the back side of the piston to provide the force to stroke the piston. A 0.055 -inch diameter 2024-T4 aluminum shear pin (80 pound-force static strength) held the pin in the extended position until the NSI was fired. A deep-drawn, energy-absorbing crushable steel cup was attached to the back side of the piston to stop the piston at the limit of its stroke. The cup, which had a 0.010-inch wall thickness and was 0.25 -inch deep, not only absorbs the excess energy of the moving piston/pin, but prevents piston impact to reduce pyrotechnic shock. Also, deformation of this cup locks the piston/pin in the withdrawn position, assuring no rebound.

\section{$\underline{\text { NASA Standard Initiator (NSI) }}$}

The NSI, shown in cross section in figure 2 and described in references 6 and 7, is an electrically initiated cartridge which was designed to produce heat, light, gas and burning particles. Three different manufacturing lots were tested: the Viking Standard Initiator (VSI), NSI lot XPJ and NSI lot XDB; the VSI and lot XPJ were from one supplier and lot XDB was from another. The major physical difference among the three lots was the $\mathrm{KClO}_{4}$ oxidizer; two different manufacturing processes were employed, yielding different particle sizes and shapes. The $\mathrm{KClO}_{4}$ used in the Viking lot and the NSI lot XDB was manufactured using a hammer mill process. That is, the $\mathrm{KClO}_{4}$ powder was pulverized by repeated impacts of steel hammers attached to the sides within a rotating cylinder. This process produced an irregularly shaped particle. The average particle size for lot XDB was 10 microns in size; the particle size for the VSI lot was much smaller. The $\mathrm{KClO}_{4}$ for NSI lot XPJ was produced by a fluid mill process, which sprayed and dried a fluid mixture in an inert gas environment. This process produced highly uniform, cylindrical particles with an average particle size of 3 microns.

\section{TESTING AND ANALYSIS PROCEDURES}

Tests were conducted to determine how: (1) the pin puller operates, (2) the NSI powers the pin puller to accomplish the required function, (3) environments effect the pin puller, and (4) the pin puller functioned in its final application. An analysis used the data collected to determine the functional margin and to predict reliability of the pin puller for the flight.

Several experimental configurations were used to accomplish these tests. To determine how the pin puller operates, the dynamic input from the NSI was simulated by dropping a weight onto the vertically oriented pin. The NSI output to the pin puller was determined by measuring the pressure produced and the energy of the moving piston/pin by crushing honeycomb and the pin puller's energy absorbing cup.

\section{Weight Drop Tests}

The pin puller mechanical performance was evaluated through measurements of force and energy by dropping 1,2 or 3-pound cylindrical weights onto the vertically oriented $\operatorname{pin}^{2,3}$. The falling weight, impacting on the pin, simulated the impulsive input of the output of the NSI. The validity of the simulation was based on comparing the function times (at comparable energy levels) that were achieved by the weight impact (1.2 milliseconds) to that achieved in actual firings (0.4 millisecond); no other method has been found that approaches this similarity, while accurately controlling input energy levels. Piezoelectric load cells were positioned under the pin puller to measure the mechanical resistive forces during the actuation of the pin puller. A magnetic tape recorder provided an overall system frequency response linear to $80 \mathrm{khz}$. The weights were guided to impact, using a tube supported by a tripod. This approach provided for a highly reproducible energy input, measured in inch-pounds (drop height multiplied by the drop weight). The drop height of the weight was reduced to the minimum level required to stroke and lock the piston to determine the "energy required" value to 
function the pin puller. Drop heights were increased to determine the effects of excess energy beyond that needed to function the pin puller. New energy absorbing cups were installed after each drop and the amount of crush was measured. Sliding friction, a key performance parameter, was controlled by lubrication.

\section{NSI Energy Output Tests}

The performance of the NSI in the pin puller was evaluated by measuring the pressure produced in the pin puller and the energy imparted into the moving piston/pin. Pressure was measured by installing a piezoelectric pressure transducer in the second NSI port. The data was recorded on the magnetic tape recorder, described above. Two different methods were used to measure energy in the moving mass of the piston/pin.

The energy delivered by NSI firings was measured by: (1) the pin puller's energy absorbing cup, and (2) aluminum honeycomb. Both the energy absorbing $\operatorname{cup}^{2}$ and the honeycomb ${ }^{3}$ exhibited a linear response to increasing energy inputs. After each test firing, the pin puller was disassembled and the amount of cup crush was measured to determine the energy delivered by the NSI in that particular test, using the weight drop test results as the calibration. This method was used as the performance standard for the reliability analysis. The aluminum honeycomb provided a comparison technique for the evaluation of the VSI and two lots of NSIs. The stroke of the piston/pin of the pin puller was transferred through an adapter piston to the precalibrated aluminum honeycomb. The honeycomb resisted the piston/pin throughout its stroke to produce an energy value in inch-pounds (crush distance multiplied by crush strength in pounds-force). A total of 5 to 10 units of each VSI/NSI lot were test-fired in this configuration. Although the honeycomb provided a useful comparative method, the energy values obtained did not represent the functioning of the pin puller in the HALOE Instrument, and thus could not be used for the reliability analyses.

\section{Environmental Effects}

Ten pin pullers were subjected to the HALOE environments expected in the flight. These tests included vibration, constant acceleration, mechanical shock and thermal/vacuum exposures. The units were $\mathrm{x}$-rayed before and after environmental exposures.
The units were functioned at laboratory ambient and under thermal/vacuum conditions. The units were disassembled and the amount of crush of the energy absorbing cups was measured to determine performance.

System Tests

Five test firings were conducted with the pin puller assembled with two NSIs and installed in the flight instrument. Only one NSI was fired. Two tests were conducted with the worst-case side loads on the pin in its mating socket. After the firings, the pin pullers were disassembled and the amount of crush of the energy absorbing cups was measured to determine how much energy was imparted into the piston/pin.

Mechanical Functional Margin and Reliability Analyses

At this point, following the experimental effort, the most significant failure-inducing variable was clearly the energy delivered by the NSI. Therefore, the analysis focused on comparing the energy required to function the pin puller to the energy delivered by the NSI.

Functional Margin. Since functional margin is the energy that is excess to that needed to accomplish the function, the following definition was used:

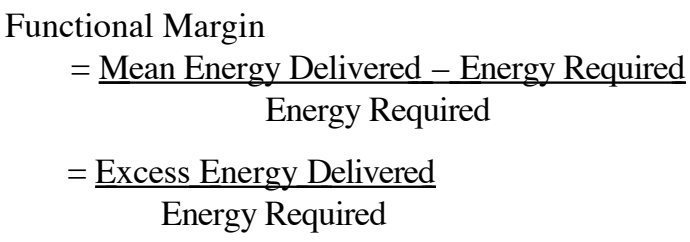

Only the five Energy Delivered data points collected in the System Test section were used. The Energy Required value was obtained from the Weight Drop Tests.

Reliability Prediction. The basis for predicting mechanical functional reliability, or the probability of success, is to analyze the most influential failureinducing variable, energy. The energy required to accomplish the desired function was compared to the energy produced by the NSI. Figure 3 shows these two parameters as Gaussian distributions, where the "Frequency of Occurrence," or the number of times a particular energy value is obtained, is plotted on the ordinate with "Increasing Energy" on the abscissa. 
Overlap of the two distributions, as shown in the lower curves, is indicative of a significant probability of failure. The top figure indicates a low probability of failure, since there is a wide separation between the two distributions and very little overlap. Clearly, to increase the probability of success, the performance distributions of a device should be as narrow (small amount of performance variation or standard deviation) and as far apart as realistically possible to reduce overlap. For example, with this assumption of Gaussian distributions and "known" means and standard deviations (sigmas), there is only a 0.00005 probability that an energy value will occur outside of the "tail" or 3.89 standard deviation. Only the upper "tail" of the Energy Required plot and the lower "tail" of the Energy Delivered" plot affect the probability of failure. Even if the distance between the 3.89 standard deviation points on these curves is zero, the product of these two probabilities yields a failure probability of less than 0.00000001 . The normal distribution curves plotted here are reasonable assumptions for the functional performance of mechanical devices, but neither the means nor standard deviations are "known." The data points collected in functional evaluations contribute to this definition, which can then be used to predict reliability using small-sample statistics. The mean and standard deviation of these data points become the "best estimates" of the actual values of the distributions. Thus the means and distributions are positioned on the experimental means. A second assumption simplifies the statistical analysis even further; the Energy Required to function a mechanism should be so well controlled that the distribution should be very sharp, or in essence, a straight line. That is, it is assumed that the Energy Required value is deterministic and can be well defined experimentally. Thus, only the lower portion of the Energy Delivered distribution contributes to a "onetail" statistical analysis.

As stated in reference 8, Part I, page 116, "Many non-statistical users of statistics are well acquainted with confidence intervals for the population mean and for the population standard deviation and some are also aware of tolerance intervals. However, very few know about prediction intervals, despite their practical importance." The "practical importance" in this case is that a one-sided prediction interval, based on a Student's " $t$ " distribution, is the simple, objective reliability prediction wanted if the distribution is normal with unknown mean and standard deviation. That is, Student's " $t$ " analyses can predict the probability of failure, or that an Energy Delivered value will be less than the Energy Required value. Figure 4 shows a $\log / \log$ plot of available " $t$ " tables, created from references 8,9 , and 10 , extrapolated to a one-in-a-million risk (probability of failure) for several sample sizes. Where $\mathrm{n}$ is equal to infinity, for example, the mean and standard deviation are "known," as described above. The ordinate, Coefficient of Sigma, a factor that establishes the prediction interval being analyzed (how many Energy Delivered standard deviations separate the Mean Energy Delivered from the Energy Required failure point), is defined as:

$$
\begin{gathered}
\text { Coefficient of Sigma } \\
=\frac{\text { Mean Energy Delivered }- \text { Energy Required }}{\text { Energy Delivered standard deviation }}
\end{gathered}
$$

The abscissa is the probability of failure; the probability of success (reliability) is plotted on the lower scale. Once the mean and standard deviation of the Energy Delivered sample are determined, the Coefficient of Sigma is calculated; the horizontal intercept of that value with the number of functional tests conducted yields the probabilities of failure and success on the abscissa. The desirable result of this analysis is to drive the solution to the left, which can be accomplished in several ways: (1) increasing the interval between the Energy Delivered mean and the Energy Required value, (2) decreasing the Energy Delivered standard deviation, and (3) increasing the number of functional tests. Notice that the sample sizes between 20 and infinity produce a relatively small probability change, which indicates that the collection of a large number of samples is subject to diminishing return. Also notice that a confidence level is not needed here. Tolerances have confidence levels; predictions do not.

\section{$\underline{\text { RESULTS }}$}

This section describes the results of the experimental and analytical efforts to predict functional reliability of the pin puller. Detailed results are presented in the same format as the Procedures section.

\section{Weight Drop Tests}

The weight drop tests revealed a considerable difference in performance between unlubricated and lubricated pin pullers. An unlubricated interface required over 100 inch-pounds to accomplish the stroke; the o-rings rolled up on their respective axes, and chunks were torn out during the stroke. 
A typical force/time plot of a 75 inch-pound drop test (a 37.5-inch drop of a 2-pound weight) on a welllubricated pin puller is shown in figure 5. Two plots of the same event are shown; the upper trace has a scale of 50 pounds-force, while the lower has a scale of 500 pounds-force. Dynamics induced considerably higher forces, as compared to static evaluations. Instead of the static-rated value of 80 pounds-force to fail the 0.055-inch diameter shear pin, an average force of 200 pounds was required. Instead of the static-rated value of 3 pounds-force of sliding friction, the force was oscillatory, averaging 21.5 poundsforce. The last high-level force indication, averaging 1,700 pounds was induced in crushing the energy absorbing cup. A reasonable accounting can thus be made of the 75 inch-pound energy input, as shown in figure 5 and table II.

\section{TABLE II. ENERGY CONSUMPTION IN A 75 INCH-POUND INPUT TO THE HALOE PIN PULLER}

\begin{tabular}{|c|c|}
\hline $\begin{array}{l}\text { Energy Con- } \\
\text { sumed } \\
\text { inch-pounds }\end{array}$ & Derivation \\
\hline 9.8 & $\begin{array}{l}\text { Fail the shear pin }(0.049 \text {-in. } \\
\text { equivalent square pin } \times 200 \mathrm{lbs})\end{array}$ \\
\hline 12.6 & $\begin{array}{l}\text { Stroke }(0.589 \text {-in. overall stroke } \times \\
21.5 \mathrm{lbs})\end{array}$ \\
\hline 51 & $\begin{array}{l}\text { Crush the energy absorbing cup } \\
(0.030 \text {-in. } \times 1,700 \mathrm{lbs})\end{array}$ \\
\hline 1.6 & $\begin{array}{l}\text { Rebound of the weight } \\
(0.8 \text {-in. } \times 2 \mathrm{lbs})\end{array}$ \\
\hline 75 inch- & Total \\
\hline
\end{tabular}

These weight drop tests provided a conservative result, since energy losses due to non-elastic deformations were ignored.

\section{NSI Energy Output and System Tests}

Table III shows the results of the performance of three different lots of NSIs, using the honeycomb crush tests.

Typical working pressures within the pin puller for one of each of the NSI lots are shown in figure 6 . The pin reached its full stroke in less than 0.5 millisecond.
TABLE III. HONEYCOMB CRUSH ENERGY MEASUREMENTS OF THREE LOTS OF NSIS.

\begin{tabular}{ccc}
\hline \hline Lot & $\begin{array}{c}\text { Manuf. } \\
\text { Date }\end{array}$ & $\begin{array}{c}\text { Average/Standard } \\
\text { Deviation } \\
\text { inch-pounds }\end{array}$ \\
\hline Viking & 1972 & $99 / 21$ \\
NSI XPJ & 1985 & $127 / 20$ \\
NSI XDB & 1988 & $53 / 49^{*}$ \\
\hline \hline
\end{tabular}

"Actual energy values collected, reference 2, were: 26, 19, 137, 31 and 54.

The energy delivered by the five functional tests of the pin puller within the HALOE Instrument were 136, 147, 176, 176 and 190 inch-pounds. This yielded a mean of 165 and a standard deviation of 22 inch-pounds. The mean was significantly less than the functional performance of the unit outside of the instrument, which averaged 185 inch-pounds ${ }^{2}$.

\section{Environmental Effects}

Following the exposure of 10 units from the flight lot to qualification environments, the energies measured in functioning these units were comparable to those obtained in previous tests on untested units.

Mechanical Functional Margin and Reliability Analyses

The most significant failure-inducing performance variable recognized from the experimental effort on the pin puller was energy, which was used for this analysis.

Functional Margin. The functional margin analysis progressed from a simple to a more complex criteria, as shown in figure 7 . The first goal of the HALOE program office was to require that the average energy produced by the NSI in the instrument to be at least 3 times that needed to function the device. The energy required to function the pin puller, as determined experimentally, was a highly reproducible value of 25 inch-pounds, which was assumed to be a deterministic value. The mean Energy Delivered value in the instrument was 165 inch-pounds. Thus, functional margin is:

$\frac{\text { Mean Energy Delivered - Energy Required }}{\text { Energy Required }}$
$=\frac{165-25}{25}=5.6$


Even considering the minimum value of Energy Delivered observed (a test in which the pin puller had been incorrectly assembled in the instrument, and the pin was bound in its retention port), functional margin is:

$$
\frac{136-25}{25}=4.4
$$

This value is well above the program's minimum Energy Delivered goal of 3.

Reliability Prediction. Figure 7 provides a pictorial representation of how the data is used. The Energy Delivered mean of 165 inch-pounds is assumed to be the mean of the normal distribution indicated. The deterministic failure point is 25 inchpounds. The coefficient of sigma for this 5-unit sample is:

$$
\begin{gathered}
\frac{\text { Energy Delivered mean - Energy Required }}{\text { Energy Delivered standard deviation }} \\
=\frac{165-25}{22}=6.33
\end{gathered}
$$

Figure 4 is used to determine the probability of failure, or the probability that 25 inch-pounds is contained within the normal distribution in figure 7 . The 6.33 coefficient of sigma line intersects the $\mathrm{N}=5$ curve to yield a probability of failure of $0.2 \%$, or a probability of success (reliability) of $99.8 \%$.

\section{CONCLUSION}

A new approach was developed to gain confidence in the mechanical functional reliability of pin pullers for a NASA Langley Research Center spacecraft instrument. The approach is based on: (1) understanding and controlling the effects of significant variables, including environments (2) determining mechanical functional margin by measuring and comparing the Energy Delivered by the energy source, in this case the NASA Standard Initiator (NSI), to the Energy Required to accomplish the function, and (3) predicting mechanical functional reliability by applying small-sample statistics to the functional margin data.

This effort produced a number of surprising results. Dynamic inputs (impacts from weight drops to simulate the output of the NSI) revealed that considerably more energy was required to achieve the function, compared to what would be expected from static measurements of shear pin strength and sliding friction. The shear pin failed at approximately 200 pounds-force, instead of 80 . Sliding friction in a lubricated pin puller was 7 times that expected under static conditions. The lack of lubrication caused a 10 -fold increase in the energy required to accomplish the function, compared to the well-lubricated interface. Special test methods were required to measure the dynamic, impulsive energy output of the NSI, the most significant failure-inducing or least controllable variable. This energy was measured by the amount of crush induced in calibrated energy absorbing cups within the pin puller at the end of the pin's stroke. Several different lots of NASA Standard Initiators (NSIs) were found to have considerable performance differences, which was most likely caused by variations in the particle size of the $\mathrm{K} / \mathrm{ClO}_{4}$ in NSI manufacturing lots. The lot with the known larger particle size exhibited the poorest combustion performance and most erratic energy delivery. The NSI manufacturing lot with the highest, mostconsistent performance was selected by the HALOE Program for flight. Environmental tests for qualification had no influence on performance. However, more energy was required to function the pin puller, when it was functioned in the instrument as compared to an uninhibited firing. This increase in energy was due to increased friction in withdrawing the pin from the spacecraft interface.

The analysis of this data revealed that the pin puller had a healthy functional margin of 5.6, based on comparing the mean Energy Delivered by the NSI in a sample of five tests in the instrument to the Energy Required to function the pin puller. A reliability prediction of $99.8 \%$ for successful operation of the pin puller was made with this same data, using a small-sample, statistical analysis. It is assumed the Energy Delivered by the NSI is a normal distribution. Thus, a Student's "t" analysis can be used to determine the probability of failure, or that the value of Energy Required to function the pin puller will be larger than the Energy Delivered. This approach provides designers a reasonable reliability analysis with 20 or fewer test units, which is within the limits normally applied for qualification of current systems.

The application of this energy-based, small-sample statistical approach is not a panacea that can be blindly applied to any pyrotechnic mechanism. Severe, but realistic, restrictions must be recognized to assure its appropriateness. The energy required to accomplish the function must be highly reproducible with a very narrow distribution of performance; if this 
value is not controlled, a considerably more complex analysis must be used than that offered here. Also, the worst case (largest energy required value recorded) should be used for the analysis. All Energy Delivered measurements should be made in the actual or a very close representation of the mechanical application. Even if the statistical distribution under evaluation is not normal, the approach recommended in this study provides far more information on which to make flight decisions, than that obtained from go/no-go statistical analyses.

\section{$\underline{\text { REFERENCES }}$}

${ }^{1}$ Bement, Laurence J. and Schimmel, Morry L.: Integration of Pyrotechnics into Aerospace Systems. Presented at the 27th Aerospace Mechanisms Symposium, May 12-14, 1993, NASA Ames Research Center, California.

${ }^{2}$ Bement, Laurence J. and Schimmel, Morry L.: Determination of Pyrotechnic Functional Margin. Presented at the 1991 SAFE Symposium, November 11-14, 1991, Las Vegas, Nevada.

${ }^{3}$ Bement, Laurence J. and Schimmel, Morry L.: A Manual on Pyrotechnic Design, Development and Qualification, NASA TM 110172, June, 1995.

${ }^{4}$ Bement, Laurence J.: Pyrotechnic System Failures: Causes and Prevention. NASA TM 100633, June, 1988.
${ }^{5}$ Multhaup, Herbert A.: Reliability Estimates from Small Samples, page 137. ISBN; 0-9639998-0-X, Proceedings of the ISSAT International Conference on Reliability \& Quality in Design, March 16-18, 1994, Seattle Washington.

${ }^{6}$ SKB2626100066, Design and Performance Specification for NSI-1 (NASA Standard Initiator-1), Revision C, March 7, 1990.

${ }^{7}$ Bement, Laurence J.; Schimmel, Morry L.; Karp, Harold and Magenot, Michael C.: Development and Demonstration of an NSI-Derived Gas Generating Cartridge (NGGC). Presented at the 1994 NASA Pyrotechnic Systems Workshop, Albuquerque, New Mexico, February 8 and 9, 1994.

${ }^{8}$ Hahn, Gerald. J.: Statistical Intervals for a Normal Population, Part I. Tables, Examples and Applications. Journal of Quality Technology, Volume 2, Number 3, July 1970, page 116. Part II. Formulas, Assumptions, some Derivations. Journal of Quality Technology, Volume 2, Number 4, October 1970, page 195.

${ }^{9}$ Hahn, Gerald. J.: Additional Factors for Calculating Prediction Intervals for Samples from a Normal Distribution. Journal of the American Statistical Association, Volume 65, Number 332, December 1970, pages 1668-1676.

${ }^{10}$ Dixon, W. J. and Massey, M. J., Jr.: Introduction to Statistical Analysis, 1979, 4th Edition, McGrawHill Book Co., pages 92, 512. 


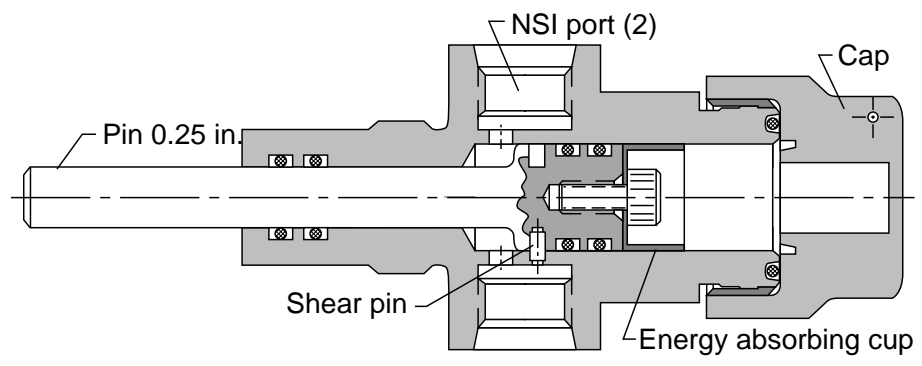

Figure 1. Cross sectional view of redesigned HALOE pin puller.

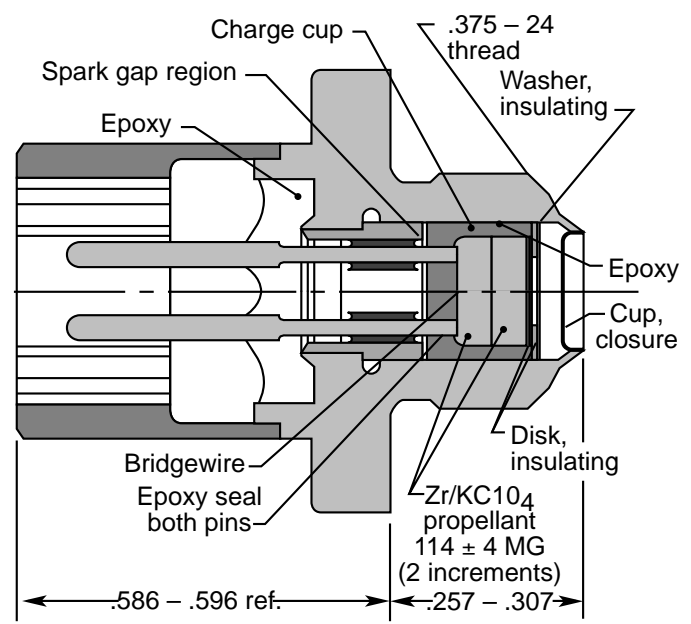

Figure 2. Cross sectional view of NASA Standard Initiator (NSI).

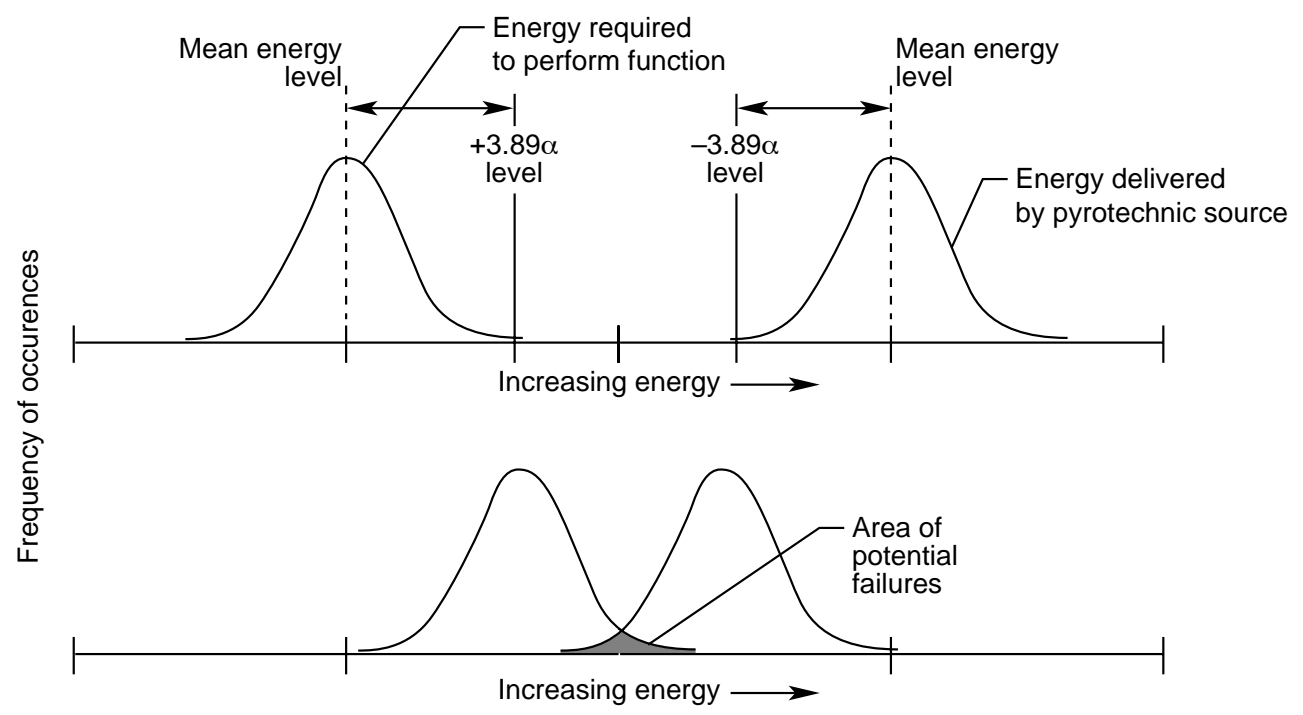

Figure 3. Statistical presentation of reliability based on comparing the Energy Delivered to the Energy Required to accomplish a desired function. The $+/-3.89$ sigma levels for each distribution contain $99.99 \%$ of the population. 


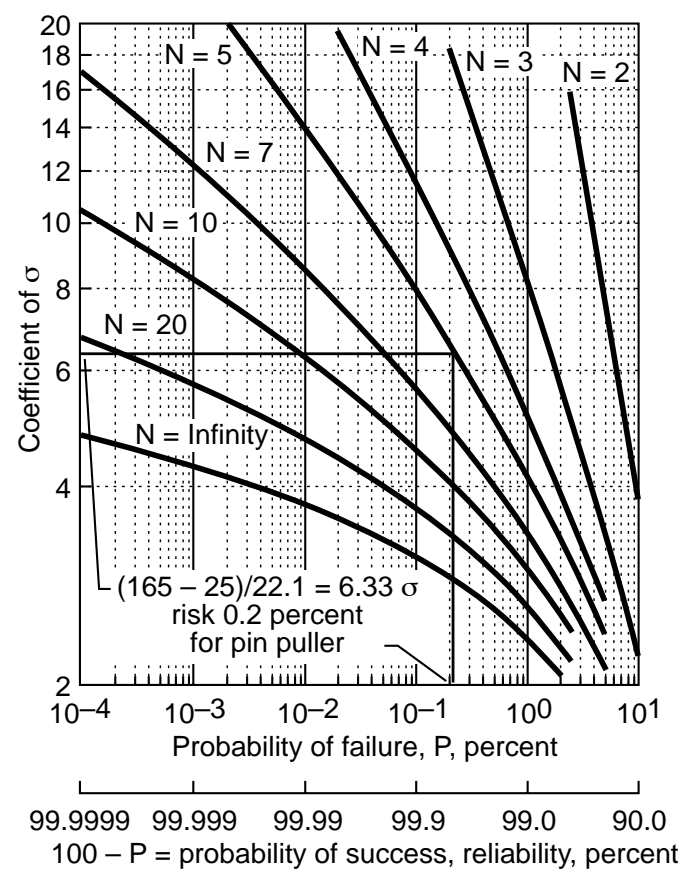

Figure 4. Statistical presentation of small-sample predictions of the risk of failure and reliability.

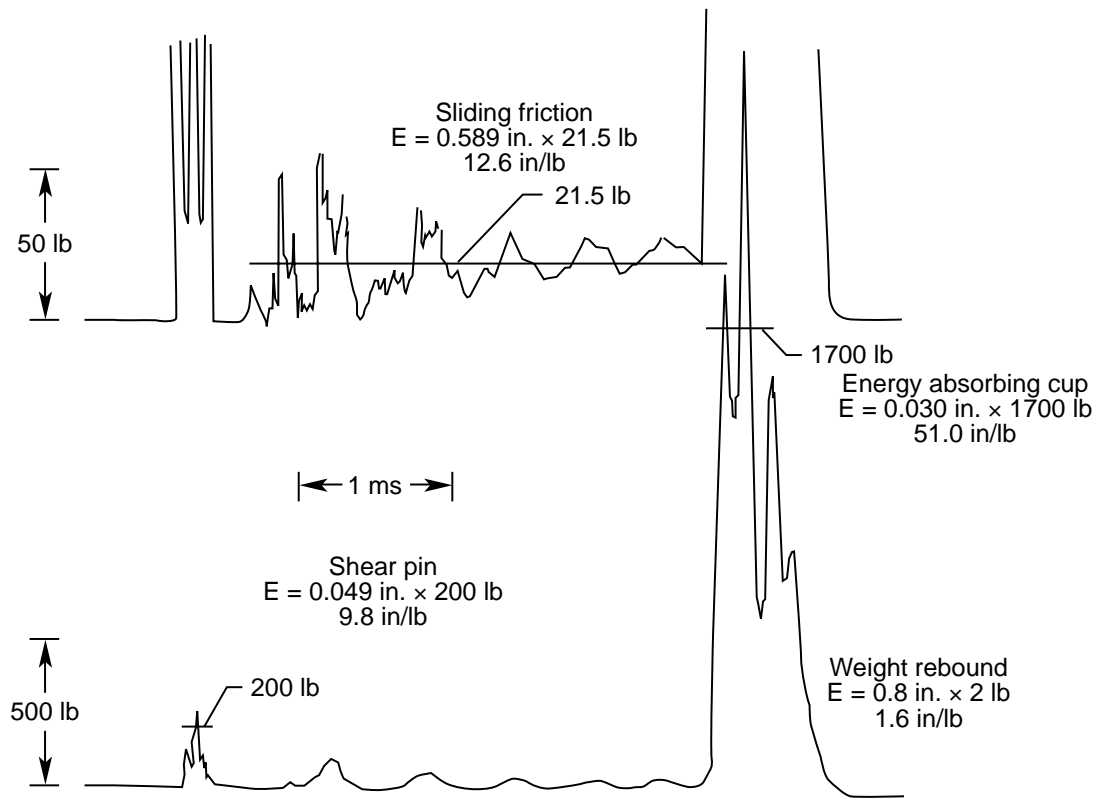

Figure 5. Force/time history (two scales/same event) of well-lubricated HALOE pin puller with 75 inch-pound weight drop test input (37.5-inch drop, 2-pound weight). 


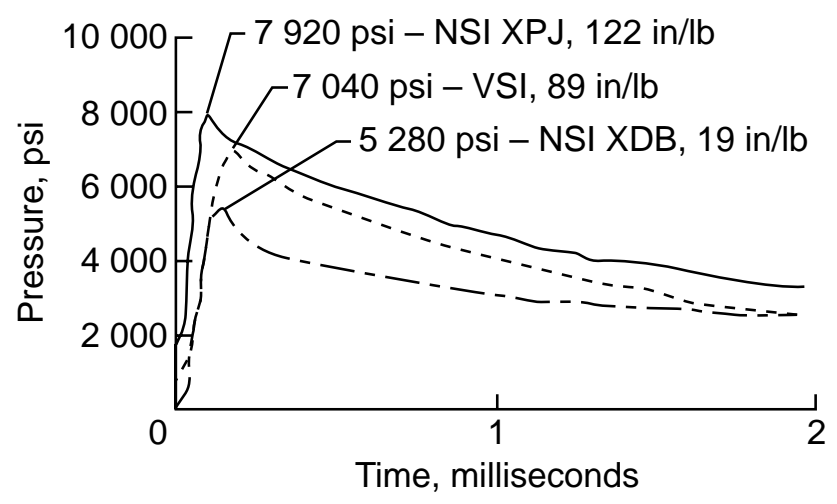

Figure 6. Typical pressure traces produced during firing of three NSI lots in the HALOE pin puller, indicating the peak pressures and energies delivered.

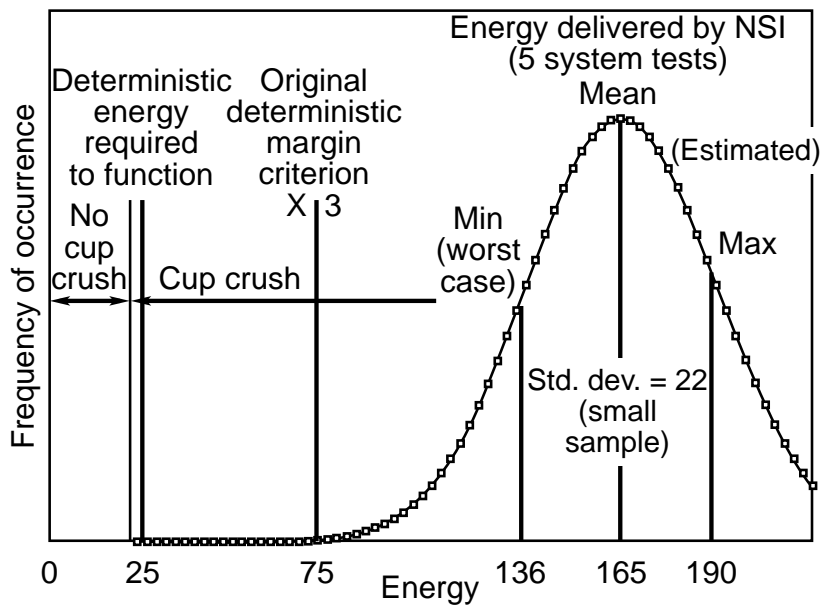

Figure 7. Statistical presentation of functional margin for the redesigned HALOE pin puller. The sample mean is the best estimate of the distribution mean. 